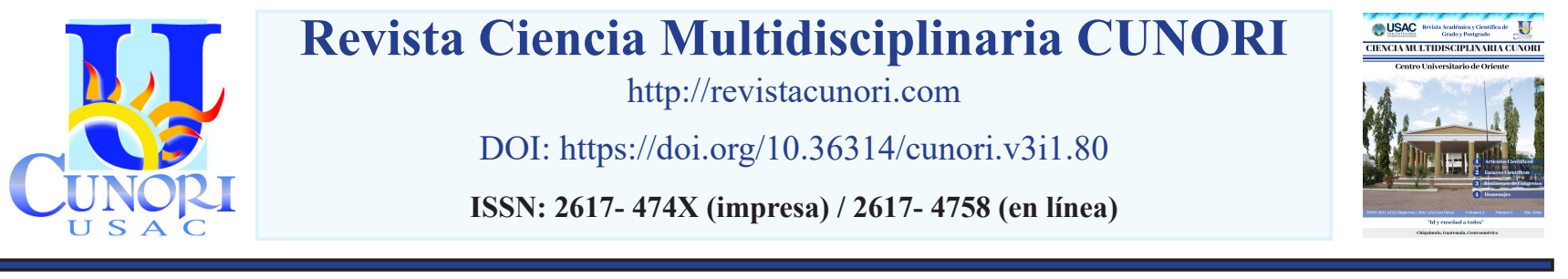

Como citar el artículo

Barrios, W., Mérida, L., Sosa, C., Arriola, C., Mazariegos., (2019). Caracterización del trauma craneoencefálico. Revista Ciencia Multidisciplinaria CUNORI, 3(1), 57-64. DOI: https://doi.org/10.36314/cunori.v3i1.80

\title{
Caracterización del trauma craneoencefálico
}

\section{Characterization of the craneoencephal trauma}

\author{
Wendy Barrios, Luis Mérida, Christian Sosa, Carlos Arriola, Edvin Mazariegos \\ Centro Universitario de Oriente (CUNORI), Universidad de San Carlos de Guatemala
}

Recibido: 17 de agosto de 2018 / Revisión: 11 de septiembre de 2018 / Aceptado: 18 de enero de 2019

Disponible en internet el 30 de agosto de 2019

Resumen

*Autor para correspondencia.

Correo electrónico: whitnylima.100pts@hotmail.com

A e denomina recurrencia al desarrollo de una nueva hernia independientemente del sitio anatómico, con la única condición de $\checkmark$ que sea del mismo lado operado previamente. El estudio realizado fue de tipo descriptivo retrospectivo, con el propósito de determinar la recurrencia de las hernias inguinales de acuerdo a las técnicas de reparación quirúrgica en los pacientes postoperados en el Servicio de Cirugía General de adultos del Hospital Regional de Zacapa entre enero de 2013 a diciembre de 2017. La información obtenida fue por medio de la ficha recolección datos, que ayudó a determinar una alta prevalencia de recurrencia de hernia inguinal con un 7\% (10) de 137 casos que fueron intervenidos por hernia inguinal, con un predominio en el sexo masculino con un $90 \%$ (9), la edad dominante fue rango de $>60$ años con $60 \%$ (6), según su cronología tiene predominio en un 100\% (10) para recurrencia tardía. Según escala de Campanellí la mayor frecuencia fue de R2 con un total de 60\% (6) de tipo indirecta; con 60\% (6) tipo directa según la escala de Nyhus y su localización anatómica con un 70\% (7) casos fue lado derecho. La técnica más utilizada en la primera intervención quirúrgica fue la de Lichtenstein en un 71\% (97) casos y la más utilizada en la primera intervención quirúrgica de hernia inguinal recurrente es la técnica de Bassini con 40\% (4), de acuerdo con otros estudios se afirma que es la que más presenta recurrencia en comparación de la técnica Lichtenstein con 10\% (1).

Palabras clave: hernia inguinal, recurrencia de hernia inguinal, técnica quirúrgica

Abstract

$\mathrm{R}$ ecurrence is called the development of a new hernia regardless of the anatomical site, with the only condition that it be from the same side previously operated. The study carried out was of a retrospective descriptive type, with the purpose of determining the recurrence of the inguinal hernias according to the surgical repair techniques in the postoperative patients in the Service of General Surgery of adults of the Regional Hospital of Zacapa between January 2013 to December 2017. The information obtained was through the data collection form, which helped determine a high prevalence of inguinal hernia recurrence with $7 \%$ (10) of 137 cases that were operated on due to inguinal hernia, with a predominance in the male sex with a $90 \%$ (9), the dominant age was range of $>60$ years with $60 \%$ (6), according to its chronology it has a predominance in $100 \%$ (10) for late recurrence. According to the Campanellí scale, the highest frequency was R2, with a total of $60 \%$ (6) of indirect type; with $60 \%$ (6) direct type according to the Nyhus scale and its anatomical location with 70\% (7) cases was right side. The technique most used in the first surgical procedure was that of Lichtenstein in 71\% (97) cases and the most used technique in the first recurrent inguinal hernia surgery is the Bassini technique with $40 \%$ (4), according to others Studies are said to be the most recurrent compared to the Lichtenstein technique with $10 \%(1)$.

Keywords: cranioencephalic trauma, characterization, Glasgow scale, brain injury 


\section{Introducción}

El trauma craneoencefálico (TCE) se define como una alteración en la función cerebral u otra evidencia de patología cerebral causada por una fuerza externa a causa de un impacto directo sobre el cráneo, aceleración o desaceleración rápida, penetración de un objeto (arma de fuego) u ondas de choque de una explosión (Estrada, et al., 2012:17).

Los traumatismos son una epidemia desatendida en los países en desarrollo. Ocasionan más de cinco millones de muertes al año, una cifra aproximadamente igual a las ocasionadas por el VIH/SIDA, la malaria y la tuberculosis combinados (Gosselin, et al., 2009:1). Diariamente se atienden en muchas emergencias de los hospitales nacionales de Guatemala pacientes con TCE, llegando a requerir tratamiento neuroquirúrgico, manejo médico en unidad de cuidados intensivos y rehabilitación.

En el 2004 Guatemala fue considerada como una de las ciudades más violetas de América Latina presentando una tasa de mortalidad por TCE que osciló entre 25-45 por 100,000 habitantes (Gámez, et al., 2013:1). Siendo esta patología observada con una elevada incidencia de morbimortalidad, debiéndose a los escasos recursos que presentan los hospitales para la atención, estabilización y recuperación del paciente traumatizado.

Por lo anterior se realizó un estudio descriptivo retrospectivo cuyo objetivo fue caracterizar al paciente con trauma craneoencefálico que fue atendido en el servicio de emergencia del Hospital Nacional de El Progreso, llenando la boleta de recolección de datos que incluyo datos generales y caracterización del TCE (género, edad, ocupación, grado del TCE, mecanismo de lesión, lesiones diagnosticadas por tomografía (TAC), complicaciones, días de estancia hospitalaria).

\section{Materiales y métodos}

Se recolectaron los datos por medio de una boleta de recolección, que consto de dos partes que incluyó: datos generales y caracterización del trauma craneoencefálico. El estudio se realizó en el área de archivo del Hospital Nacional de El Progreso, Guatemala. Se llenó la boleta de recolección respectivamente por cada expediente. Luego de haber alcanzado el total de la muestra, se obtuvo la información de la boleta recolectora, se tabularon los datos recopilados. 


\section{Resultados}

Edad de los pacientes con diagnóstico de trauma craneoencefálico

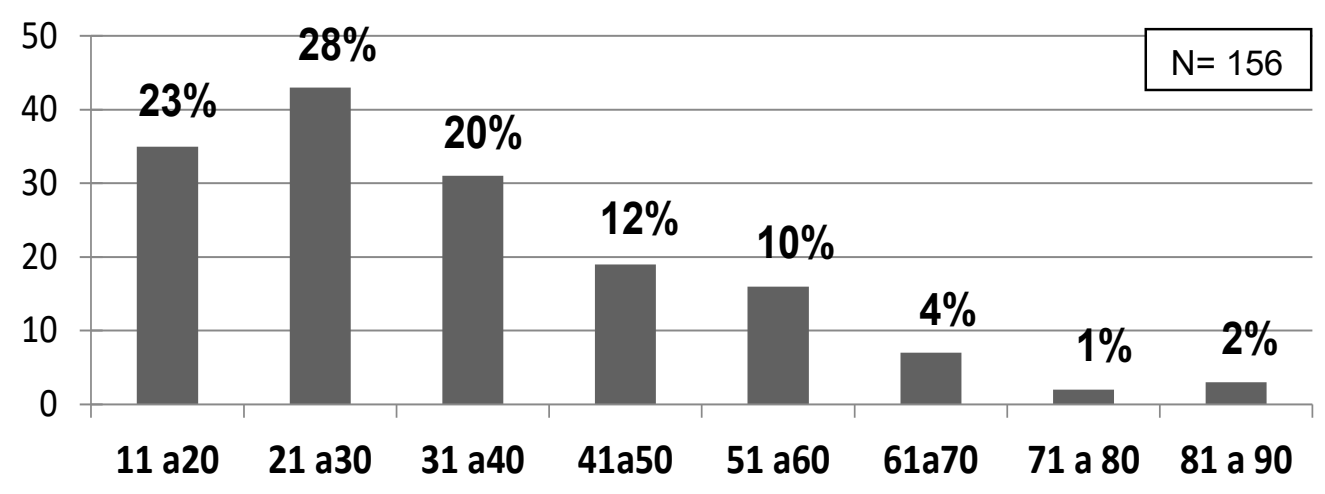

Figura 1. Distribución de pacientes por grupo etario con trauma craneoencefálico atendidos en la emergencia del Hospital Nacional de El Progreso durante enero 2013 a diciembre 2017.

La distribución del rango por edades la cual fue de la siguiente manera: $28 \%$ (43) de los pacientes quedaron en el rango de 21 a 30 años, el 23\% (28) en el rango de 11 a 20 años, un 20\% (27) quedaron en el rango de 31 a 40 años, $12 \%$ (24) en el rango de 41 a 50 años.

\section{Género más afectado}

$N=156$

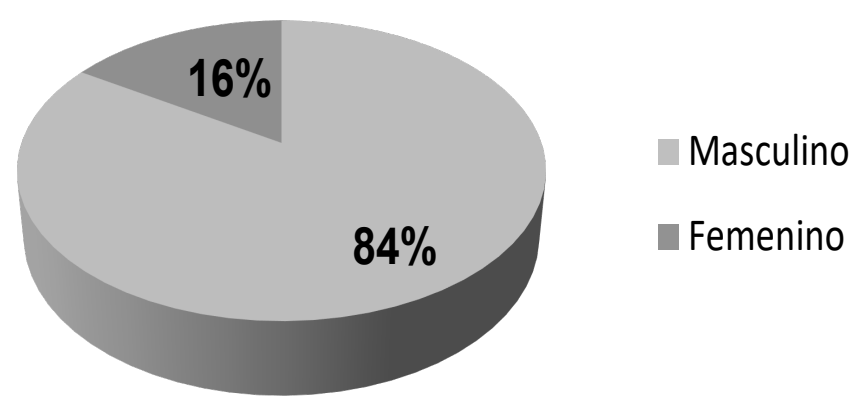

Figura 2. Distribución de pacientes por género con trauma craneoencefálico (TCE) atendidos en la emergencia del Hospital Nacional de El Progreso durante enero 2013 a diciembre 2017.

De los pacientes que sufrieron trauma craneoencefálico según el género predominó con el 84\% (131) el sexo masculino y solo el 16\% (25) son del género femenino. 


\section{Mecanismo de lesión}

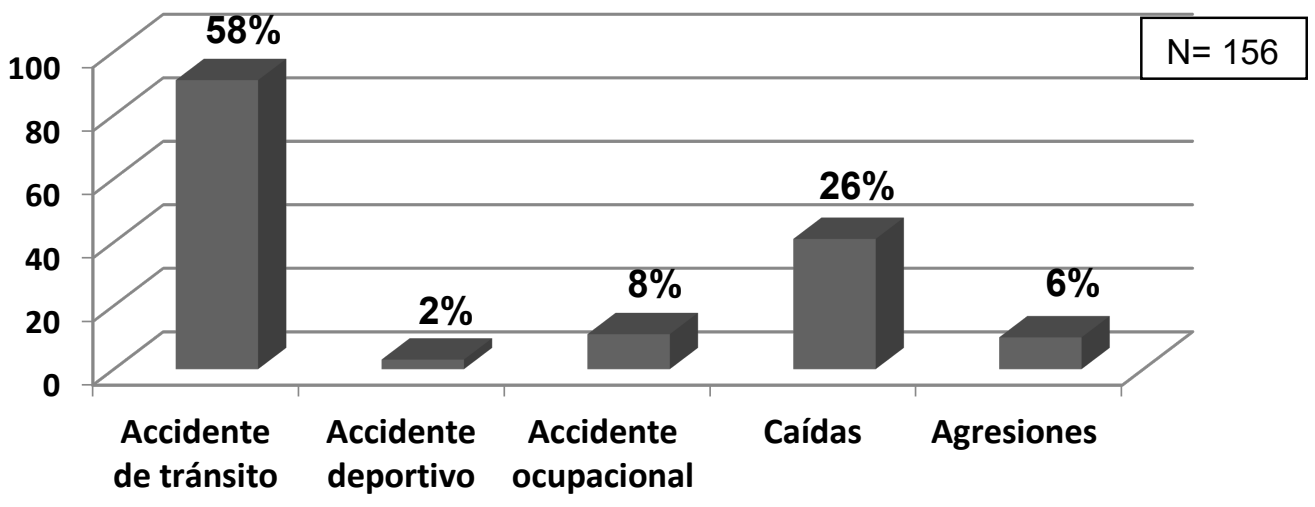

Figura 3. Distribución de pacientes por causa del trauma craneoencefálico atendidos en la emergencia del Hospital Nacional de El Progreso durante enero 2013 a diciembre 2017.

Se encontró que la causa más frecuente de trauma craneoencefálico fue $58 \%(91)$ de los pacientes por accidente de tránsito, seguidas por 26\% (41) caídas, $8 \%$ (11) por accidente ocupacional, $6 \%$ (10) por agresiones y solamente el $2 \%$ (3) fueron acci dente deportivo.

\section{Según la severidad del trauma craneoencefálico}

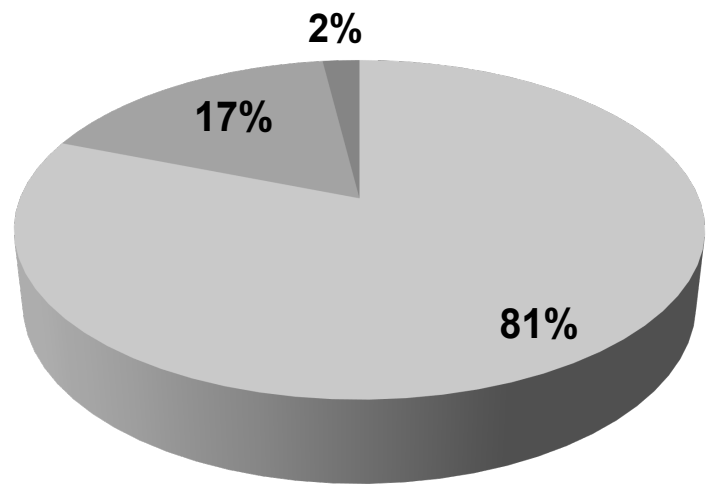

$\mathrm{N}=120$

Figura 4. Distribución de pacientes por grado de trauma craneoencefálico atendidos en la emergencia del Hospital Nacional de El Progreso durante enero 2013 a diciembre 2017.

En esta grafica se observa que el 81\% (97) de los pacientes tuvieron TCE grado I, el 17\% (20) tuvieron grado II y solo el 2\% (3) tuvieron grado III. 


\section{Pacientes que se realizaron Tomografía Axial Computarizada}

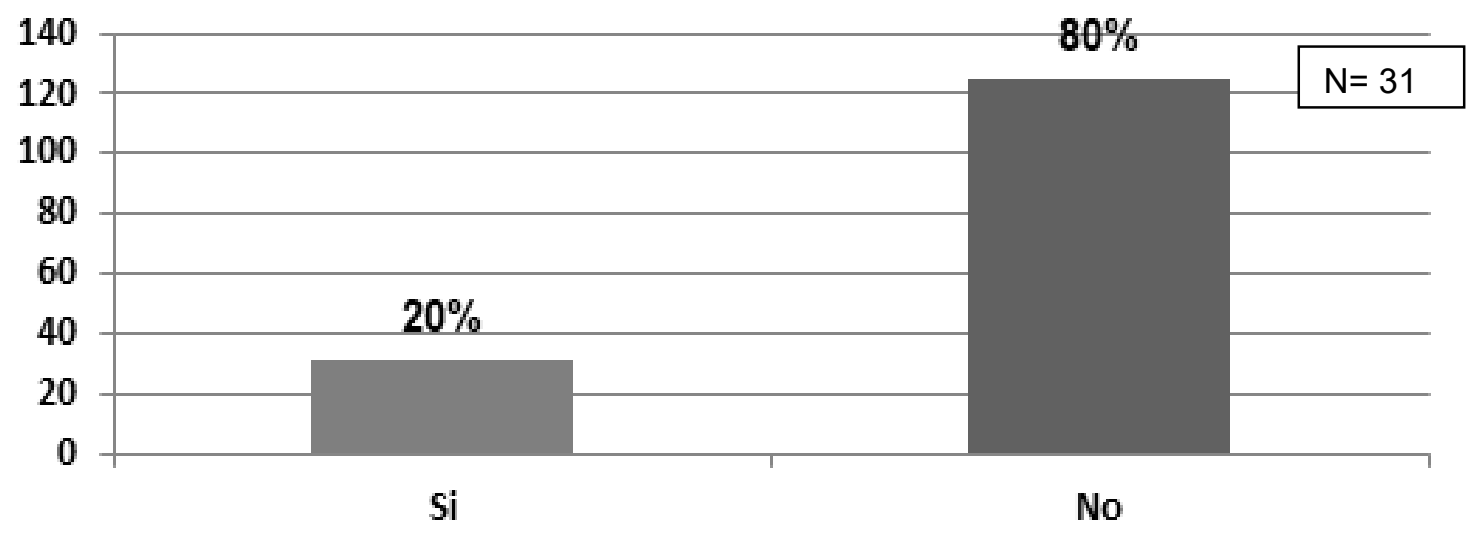

Figura 5. Distribución de pacientes que se realizaron Tomografía Axial Computarizadaque sufrieron trauma craneoencefálico atendidos en la emergencia del Hospital Nacional de El Progreso durante enero 2013 a diciembre 2017.

Se obtuvo que solamente un $20 \%$ de los pacientes se realizaron Tomografía Axial Computarizada y el $80 \%$ no se realizó.

\section{Utilización de la Escala de Coma Glasgow}

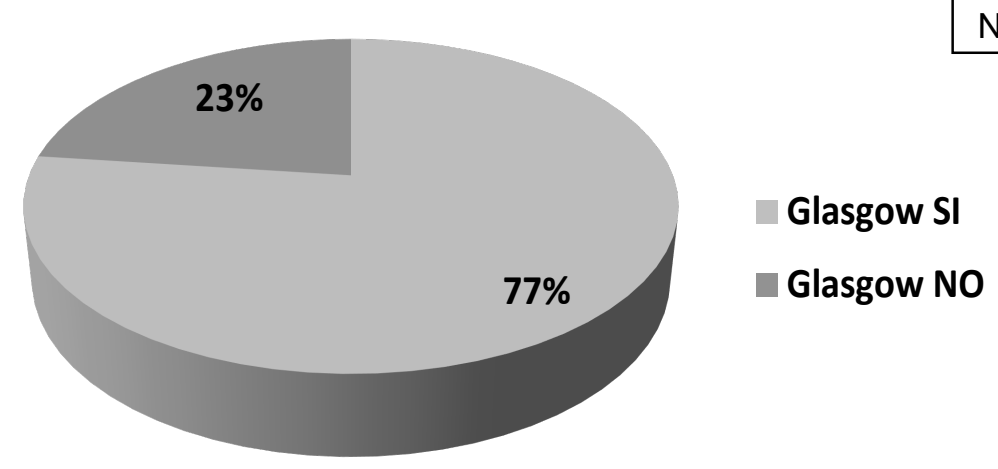

Figura 6. Distribución de pacientes con trauma craneoencefálico a quienes se les realizó escala de Glasgow atendidos en el Hospital Nacional de El Progreso durante enero 2013 a diciembre 2017.

La distribución de los pacientes según si se realizó o no la escala de Glasgow fue de la siguiente manera: un $77 \%$ (120) se le realizó la escala y a un 23\% (36) no se le realizó. 


\section{Complicaciones, intervenciones y ventilación asistida}

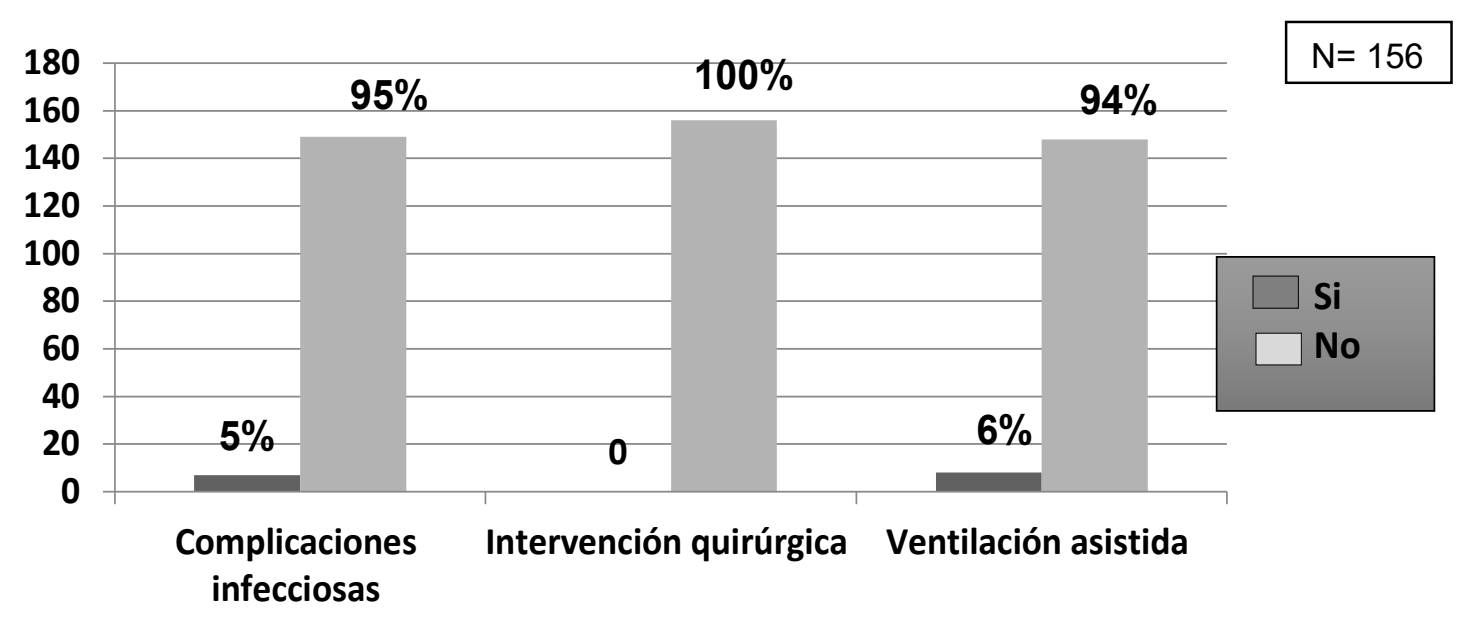

Figura 7. Distribución de pacientes con trauma craneoencefálico por complicaciones infecciosas, intervenciones quirúrgicas y necesidad de ventilación asistida atendidos en la emergencia del Hospital Nacional de El Progreso durante enero 2013 a diciembre

En la presente gráfica se hace notar que un 5\% (7) de los pacientes tuvo complicaciones infecciosas, ninguno fue sometido a procedimientos quirúrgicos y $6 \%$ (8) fueron atendidos con ventilación asistida.

\section{Tasa de mortalidad}

No. de defunciones registradas

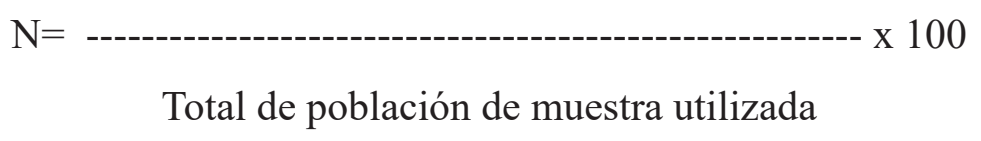

5

$$
\mathrm{N}=\text {-------- x } 100=3 \%
$$

De los 156 pacientes en estudio, se contabilizaron $5(3 \%)$ defunciones, enfatizando al decir que todos fallecieron al arribo de la emergencia del Hospital Nacional de El Progreso.

\section{Discusión}

Los pacientes jóvenes-adultos presentaron mayor vulnerabilidad de sufrir un trauma craneoencefálico ya que los resultados muestran que el $28 \%$ de los pacientes se encuentran entre edades de 21 a 30 años, seguido por el $23 \%$ en el rango de 11 a 20 años. Es de suma importancia mencionar que existe una marcada diferencia en cuanto al género más afectado, ya que se evidencio que el $84 \%$ de los pacientes que sufrieron trauma craneoencefálico pertenece al género masculino. La mayor incidencia obtenida en varones puede ser el resultado de la práctica de actividades de mayo riesgo laboral y un mayor número de lesiones relacionadas con la violencia en comparación con las mujeres (Teasell, et al., 2007:4). 
Se comprobó que la primera causa de trauma craneoencefálico de los pacientes que consultaron al Hospital Nacional de El Progreso son los accidentes de tránsito 58\%, según la severidad de trauma, se encontró que la mayor parte de los pacientes $81 \%$ presentaron TCE leve, el 17\% TCE moderado y únicamente el $2 \%$ con TCE severo. De estos pacientes, solamente el 20\% si se realizaron tomografía axial computarizada cerebral, el resto no pudo realizarse dicho estudio por los escasos recursos económicos y por no estar disponible en el hospital u otros centros asistenciales cercanos dicho estudio de imagen.

Así mismo es de suma importancia resaltar que únicamente al 77\% de los pacientes que entraron en estudio se les aplicó la escala de Glasgow, debido a que el 23\% de los pacientes llegaron con etilismo agudo al servicio de emergencia, lo cual alteraba el uso de la escala, cabe mencionar que a ningún paciente se le realizó intervención neuroquirúrgica ya que en este centro hospitalario no se cuenta con equipo adecuado, neurocirujano ni con unidad de cuidados intensivos para la realización del abordaje de estos pacientes que ameritaban la intervención especializada. En cuanto a la tasa de mortalidad que se obtuvo en el estudio fue del 3\% de los pacientes, cabe resaltar que fallecieron al llegar al servicio de emergencia del Hospital Nacional de El Progreso.

\section{Agradecimientos}

Este estudio se realizó mediante el apoyo de los asesores Dr. Luis Fernando Mérida Molina y el Ing. Christian Edwin Sosa Sancé, así mismo por el Coordinador de la Carrera de Médico y Cirujano como de los revisores de tesis. Se agradece la colaboración del personal de estadística, archivo y del director del Hospital Nacional de El Progreso por brindar su apoyo al estudio.

\section{Referencias bibliográficas}

Estrada Rojo, F., Morales Gomez, J., Tabla Ramon, E., Solis Luna, B., Navarro Argüelles, H., Martinez Vargas, M., . . . Navarro, L. (2012). Neuroprotección y traumatismo craneoencefálico. Revista de la Facultad de Medicina UNAM, 16-29. Recuperado de http://www.medigraphic.com/pdfs/facmed/ un-2012/un124d.pdf

Gámez Urizar, N.E; Cano Hernández, L.P; Reyes Donis, C.A.S; Tebelán Lec, Y.E, Ruiz Marroquin, J.M; Roque Sosa, P.F. (2013.) Caracterización epidemiológica del paciente con trauma craneoencefálico (en línea). (Tesis Lic, USAC, Guatemala.) Recuperado de http://biblioteca.usac.edu.gt/tesis/ 05/05_9197.pdf

Gosselin, R.A; Spiegel, D.A; Coughlin, R; Zirkle, L.G. (2009.) Los traumatismos: el problema sanitario desatendido en los países en desarrollo (en línea). Boletín OMS 2009-87: 245-324. Recuperado de http://www.who.int/bulletin/volumes/87/4/08-052290/es/

Teasell, R., Aubut, J., Bayley, M., \& Cullen, N. (2007). Epidemiologia y resultados a largo plazo de las lesiones cerebrales adquiridas. Revista Fundación MAPFRE, 12. Recuperado de http:/www.traumatismocraneoencefalico.com/doc/modulo2.pdf 


\section{Sobre la autora}

\section{Wendy Elizabeth Barrios Lima}

Médico y Cirujano, egresada del Centro Universitario de Oriente, CUNORI, de la Universidad de San Carlos de Guatemala en el año 2018. En el mismo año obtuvo una Especialización en Investigación a nivel de Postgrado, en el mencionado Centro Universitario. Ha realizado las siguientes investigaciones: Uso de la infusión del tabaco para la erradicación de pediculus humanus var capitis (2012).Tomografía por emisión de positrones en la actualidad (2013). Prevalencia de Insuficiencia Renal Crónica en pacientes adultos con enfermedades crónicas subyacentes del Hospital Regional de Zacapa (2015). Trauma de abdomen cerrado (2015). Evaluación del estado nutricional, el índice de masa corporal y la circunferencia abdominal como factor de riesgo cardiovascular (2015). Relación de la circunferencia cefálica con microcefalia y Zika. (2016). Pacientes con NIC I que evolucionaron a NIC II luego del tratamiento con crioterapia en la clínica de colposcopia del Hospital Regional de Zacapa (2016). Papanicolaou falsos positivos versus falsos negativos (2017). Caracterización de pacientes con insuficiencia renal crónica en Aldea el Sauce (2017).

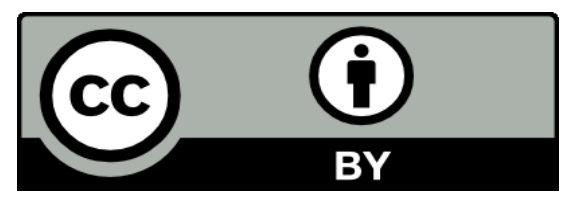

Este texto está protegido por una licencia CreativeCommons 4.0.

Usted es libre para compartir, copiar y redistribuir el material en cualquier medio o formato y adaptar el documento, remezclar, transformar y crear a partir del material para cualquier propósito, incluso comercialmente, siempre que cumpla la condición de atribución: usted debe reconocer el crédito de una obra de manera adecuada, proporcionar un enlace a la licencia, e indicar si se han realizado cambios. Puede hacerlo en cualquier forma razonable, pero no de forma tal que sugiera que tiene el apoyo del licenciante o lo recibe por el uso que hace. 